01.1;08.3;09.4

\title{
Лазерная генерация в микрорезонаторах с таммовским плазмоном и внутрирезонаторными металлическими контактами с органической активной областью
}

\author{
(C) К.М. Морозов ${ }^{1}$, А.В. Белоновский ${ }^{1,2}$, Е.И. Гиршова ${ }^{1,2}$, В.В. Николаев ${ }^{2}$ \\ ${ }^{1}$ Университет ИТМО, Санкт-Петербург, Россия \\ ${ }^{2}$ Санкт-Петербургский национальный исследовательский Академический университет им. Ж.И. Алфёрова РАН, \\ Санкт-Петербург, Россия \\ E-mail: morzconst@gmail.com
}

Поступило в Редакцию 10 декабря 2021 г.

В окончательной редакции 13 января 2022 г.

Принято к публикации 13 января 2022 г.

\begin{abstract}
Предложены конструкции вертикально-излучающего лазера с активной областью из органического светоизлучающего материала 4,4'-bis[4-(di-p-tolylamino)styryl] biphenyl с внутрирезонаторными металлическими контактами двух типов. В конструкции первого типа используются два брэгговских зеркала и два тонких металлических слоя, примыкающих к активной области. В конструкции второго типа используется одно брэгговское зеркало с тонким металлическим слоем, а в качестве второго зеркала используется толстый слой металла. Рассчитаны модовая структура и пространственное распределение оптических полей, коэффициент Парселла, а также зависимость интенсивности излучения от накачки.
\end{abstract}

Ключевые слова: таммовский плазмон, органический полупроводник, вертикально-излучающий лазер, экситон.

DOI: 10.21883/PJTF.2022.07.52285.19104

Вертикально-излучающие лазеры (ВИЛ) [1,2] находят широкое применение в различных областях науки и техники. Одна из проблем, ограничивающих эффективность ВИЛ, - проблема электрической накачки активной области, возможным решением которой является использование полупроводниковых [3] или металлических [4] внутрирезонаторных контактов. В последние годы интенсивно развиваются исследования ВИЛ с органической активной областью $[5,6]$.

В настоящей работе представлено сравнение двух типов микрорезонаторов с внутрирезонаторными металлическими контактами с органическим светоизлучающим материалом DPAVBi (4,4'-bis[4(di-p-tolylamino)styryl]biphenyl) [7] в качестве активной области.

Рассмотрим два типа микрорезонаторов с внутрирезонаторными контактами: микрорезонатор, состоящий из двух брэгговских отражателей (БО) (тип I), и микрорезонатор с одним БО и одним толстым металлическим зеркалом (тип II) (рис. 1,a). БО состоит из пяти пар $\mathrm{SiO}_{2}(81 \mathrm{~nm}) / \mathrm{Ta}_{2} \mathrm{O}_{5}(55 \mathrm{~nm})$. Оба типа резонаторов благодаря внутрирезонаторным металлическим слоям могут обеспечить электрическую накачку органического слоя [4]. Толщина внутрирезонаторного серебряного слоя составляет $40 \mathrm{~nm}$, толщина толстого металлического зеркала $616 \mathrm{~nm}$, толщина слоя органического материала DPAVBi $55 \mathrm{~nm}$. Динамика затухания фотолюминесценции (ФЛ) материала и схема молекулы показаны на рис. $1, b$. В структуре типа I существуют три локализованные оптические моды: две моды таммов- ского плазмона, локализованные на границе металла и БО, а также мода Фабри-Перо, локализованная между двумя слоями металла. Резонатор типа II рассмотрен в качестве альтернативного дизайна структуры с заменой одного БО на толстый серебряный слой. В подобном резонаторе имеет место взаимодействие двух оптических мод (мода таммовского плазмона и мода Фабри-Перо). Дисперсии собственных оптических состояний, полученные с помощью аппроксимации спектров отражения (рассчитанных с помощью метода матриц переноса), представлены для обоих типов резонаторов на рис. 2. На рисунке представлены также результаты расчета модового фактора Парселла, полученные с помощью метода $S$-квантования [8]. Дизайн микрорезонаторов был разработан исходя из того, что пик ФЛ материала DPAVBi приходится на энергию $2.6 \mathrm{eV}$, а пик поглощения материала имеет энергию $3 \mathrm{eV}$. Таким образом, в рассмотренных резонаторах одна мода может генерировать излучение, а другая может использоваться для эффективной оптической накачки.

Влияние двух рассматриваемых типов микрорезонаторов на лазерную генерацию было проанализировано с помощью скоростных уравнений. В рассмотренной модели считается, что оптическая накачка структур происходит через БО лазером, излучающим на $3 \mathrm{eV}$ с углом падения $60^{\circ}$ (область, выделенная эллипсом на рис. 2). В свою очередь излучение от структур происходит по нормали с энергиями $2.52 \mathrm{eV}$ (тип I) и $2.56 \mathrm{eV}$ (тип II). Динамическое поведение можно описать системой уравнений [9] для концентрации экситонов в верхнем виб- 


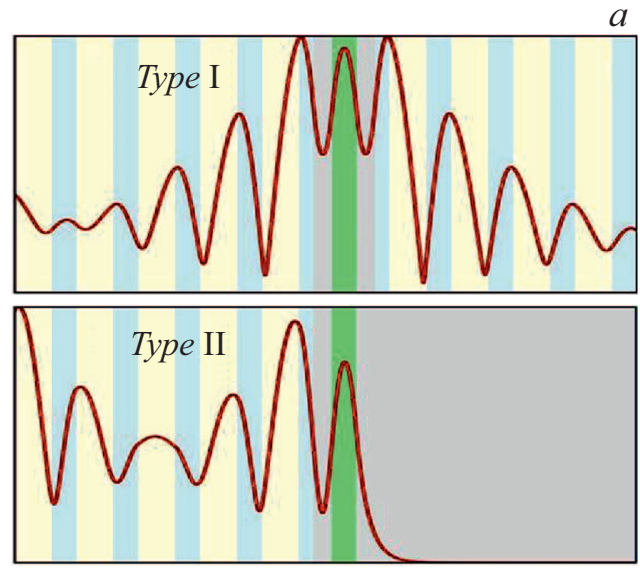

Ag $\square$ DPAVBi $\square \mathrm{SiO}_{2} \square \mathrm{Ta}_{2} \mathrm{O}_{5}$

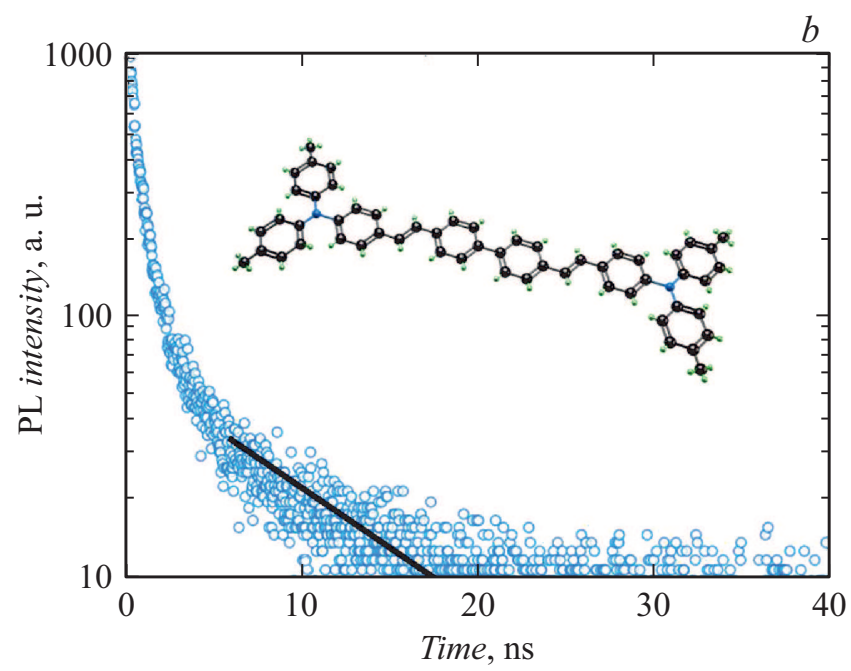

Рис. 1. $a$ - схема микрорезонаторов с внутриметаллическими слоями. Сплошной линией представлены распределения электрического поля собственной моды резонатора типа I (с энергией $2.52 \mathrm{eV})$ и типа II (с энергией $2.56 \mathrm{eV})$ для TE-поляризованной волны. $b$ - динамика затухания фотолюминесценции слоя DPAVBi толщиной $60 \mathrm{~nm}$. На вставке - структура молекулы DPAVBi. Цветной вариант рисунка представлен в электронной версии статьи.
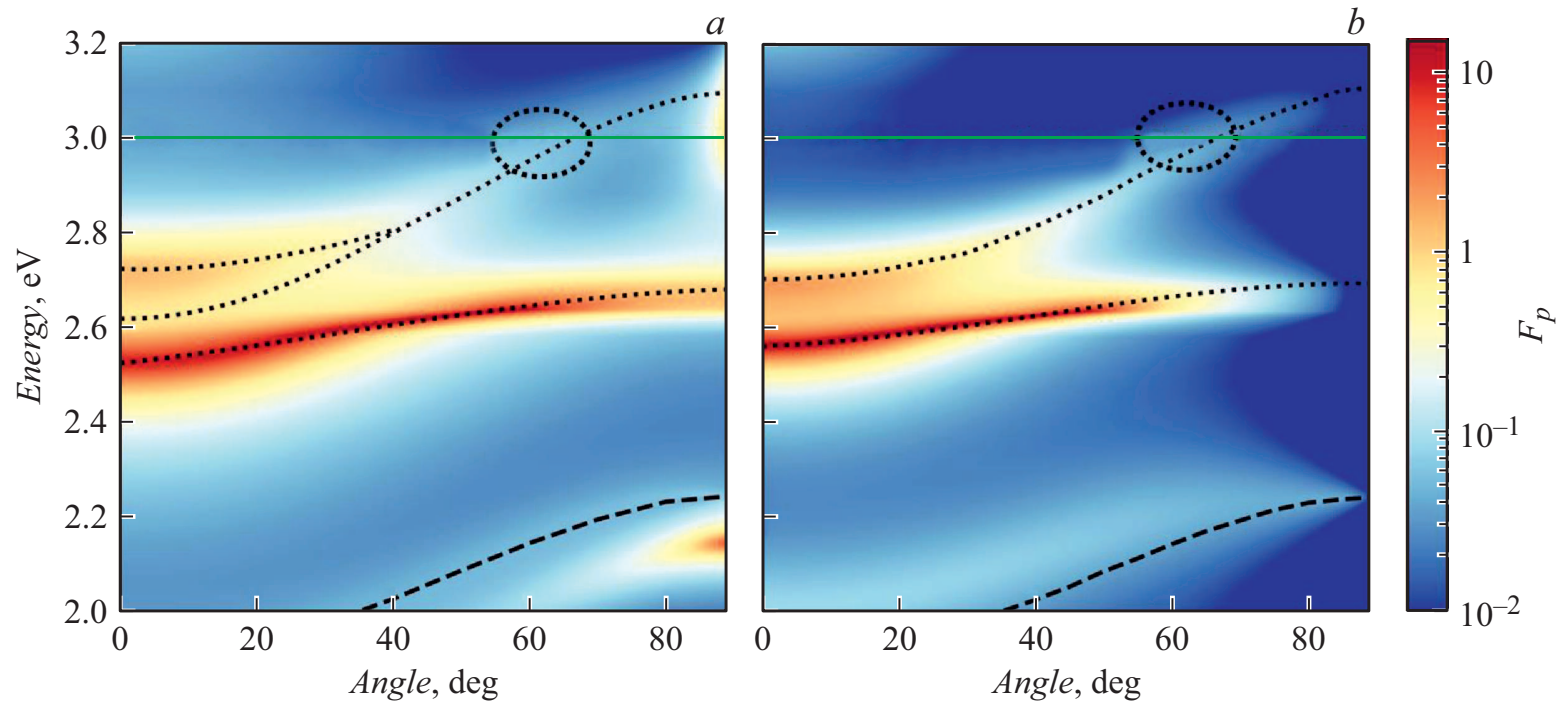

Рис. 2. Распределения модового фактора Парселла $F_{p}$ по энергии и углу падения для микрорезонатора с внутриметаллическими слоями (тип I) (a) и микрорезонатора с внутриметаллическим слоем и металлическим зеркалом (тип II) (b) для случая ТЕполяризации и диполя, помещенного в центр слоя DPAVBi. Штриховая линия - дисперсия краевого состояния БО, пунктирные линии - дисперсии мод резонаторов. Горизонтальная сплошная линия - положение пика поглощения DPAVBi. Эллипс демонстрирует область углов падения и энергий, в которой происходит накачка структур. Цветной вариант рисунка представлен в электронной версии статьи.

рационном подуровне возбужденного состояния $\left(N_{e x c}\right)$, нижнем энергетическом вибрационном подуровне $\left(N_{g r}\right)$ и концентрации фотонов в моде резонатора $(S)$ :

$$
\begin{aligned}
\frac{d N_{e x c}(t)}{d t}= & A \frac{P_{\text {pump }}}{\hbar \omega_{P}} \frac{1}{V_{P}}-k_{r e l} N_{e x c}(t)-k_{n r} N_{e x c}(t), \\
\frac{d N_{g r}(t)}{d t} & =k_{r e l} N_{e x c}(t)-\left(F_{p} k_{r}+k_{n r}\right) N_{g r}(t) \\
& -\sigma_{g} v_{g r} S(t) N_{g r}(t),
\end{aligned}
$$

$$
\frac{d S(t)}{d t}=F_{p} \Gamma \beta k_{r} N_{g r}(t)+\Gamma \sigma_{g} v_{g r} S(t) N_{g r}(t)-k_{c a v} S(t)
$$

В уравнении (1a) первый член описывает оптическую накачку с мощностью $P_{\text {ритр }}$ и энергией $\hbar \omega_{P}=3 \mathrm{eV}$, где $A-$ коэффициент поглощения структуры для угла падения $60^{\circ}$, а $V_{P}$ - объем области структуры (задается толщиной активной области $d_{c a v}=55 \mathrm{~nm}$ и радиусом пятна возбуждающего лазера $\left.a_{\text {las }}=25 \mu \mathrm{m}\right)$. Второе и третье слагаемые описывают колебательную релаксацию 

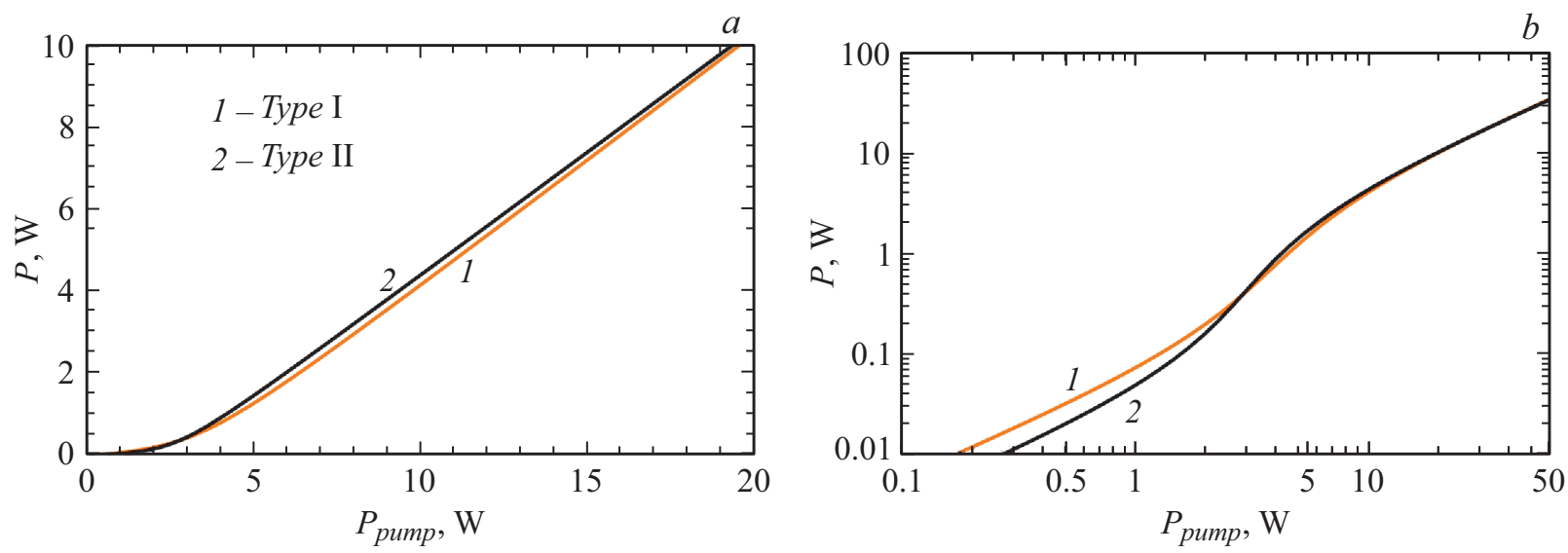

Рис. 3. Зависимость выходной мощности от мощности накачки для двух типов микрорезонаторов в линейном $(a)$ и двойном логарифмическом $(b)$ масштабах.

экситонов в низкоэнергетическое возбужденное состояние и нерадиационный уход с темпами $k_{r e l}$ и $k_{n r}$ соответственно. Скорость релаксации имеет в подобных органических материалах значения порядка $10^{15} \mathrm{~s}^{-1}$. Для данного уровня не учитывается радиационное затухание, поскольку превалирующее большинство излучательных переходов происходит из нижнего энергетического вибрационного подуровня.

Уравнение (1b) описывает динамику концентрации возбужденных состояний в нижнем энергетическом вибрационном подуровне. Второе слагаемое в нем имеет смысл спонтанного излучательного затухания возбужденного состояния с темпом $k_{r}$, которое усилено значением фактора Парселла $F_{p}$, а также нерадиационного ухода с темпом $k_{n r}$ (который совпадает с аналогичной величиной в уравнении (1a)). Третье слагаемое стимулированное излучение, определяемое следующими величинами: сечением стимулированного излучения $\sigma_{g}$ (имеющим характерное значение $4 \cdot 10^{-16} \mathrm{~cm}^{2}$ ) и групповой скоростью света $v_{g r}=c / n$.

Последнее уравнение описывает концентрацию фотонов в оптической моде структуры. Первые два слагаемых описывают приход фотонов вследствие спонтанного и стимулированного излучения соответственно. Параметр $\Gamma$ - фактор оптического ограничения, $\beta$ - вклад спонтанной эмиссии в оптическую моду, $k_{\text {cav }}=\omega / Q-$ скорость ухода фотонов из резонатора $(Q-$ добротность оптической моды).

Скорости нерадиационных процессов связаны с темпом радиационных переходов $\left(k_{r}\right)$ и внутренним квантовым выходом $(\Phi)$ соотношением $\Phi=k_{r} /\left(k_{r}+k_{n r}\right)$. В свою очередь время жизни ФЛ можно связать с темпами радиационных и нерадиационных переходов как $\tau_{\mathrm{PL}}=1 /\left(k_{r}+k_{n r}\right)$. Оценивая квантовый выход люминесценции материала в $25 \%$ и определив из экспериментального спектра затухания ФЛ (рис. $1, b)$ время жизни $\tau_{\mathrm{PL}}=9.5 \mathrm{~ns}$, можно получить следующие значения: $1 / k_{r}=38$ ns и $1 / k_{n r}=12.6 \mathrm{~ns}$.
Параметры исследуемых резонаторных структур, необходимые для решения системы (1a)-(1c), были получены с помощью метода матриц переноса (коэффициент поглощения $A$ ) и метода $S$-квантования (фактор Парселла $F_{p}$ ). Распределения электрических полей мод, локализованных в активной области (представлены на рис. $1, a)$, использовались для расчета фактора оптического ограничения $\Gamma=\int_{0}^{d}|E|^{2} d x / \int_{0}^{\infty}|E|^{2} d x$. Вклад спонтанной эмиссии в моды $\beta$ был рассчитан с использованием экспериментального спектра излучения DPAVBi [7] так, как показано в работе [10].

Система уравнений (1a)-(1c) решалась численно в квазистационарном приближении $\left(d N_{e x c}(t) / d t=0\right.$, $\left.d N_{g r}(t) / d t=0, d S(t) / d t=0\right)$ для нахождения зависимости выходной мощности, определяющейся через концентрацию фотонов в оптической моде как $P=k_{c a v} \hbar \omega S V / \Gamma$, от мощности накачки структуры $P_{\text {pump }}$. Параметрами, различающимися в двух типах резонаторов, влияющими на зависимость выходной мощности, являются $\Gamma, \beta, Q, F_{p}$ и $A$. Расчет данных параметров для первого типа резонатора дал значения $\Gamma^{\mathrm{I}}=0.0824$, $\beta^{\mathrm{I}}=0.12, Q^{\mathrm{I}}=36.7, F_{p}^{\mathrm{I}}=9.38, A^{\mathrm{I}}=0.73$, для второго типа - значения $\Gamma^{\mathrm{II}}=0.0875, \beta^{\mathrm{II}}=0.07, Q^{\mathrm{II}}=56.7$, $F_{p}^{\mathrm{II}}=12, A^{\mathrm{II}}=0.7$. Результаты расчета представлены на рис. 3. Из расчета были получены пороговые мощности накачки $\left(P_{t h}^{\mathrm{I}}=3.3 \mathrm{~W}, P_{t h}^{\mathrm{II}}=3 \mathrm{~W}\right)$ и дифференциальные эффективности $\left(\eta^{\mathrm{I}}=\frac{d P_{\text {out }}}{d P_{\text {in }}}=0.613, \eta^{\mathrm{II}}=\frac{d P_{\text {out }}}{d P_{\text {in }}}=0.597\right)$ двух рассмотренных типов резонаторов.

Рассмотренные структуры демонстрируют близкие параметры дифференциальной эффективности. Структура типа II имеет меньшие пороговые значения ввиду большей добротности собственной моды, которая в свою очередь обусловлена бо́льшим коэффициентом отражения металлического зеркала. Выбранные параметры структуры позволяют подавить поглощение излучения в металлических элементах. Сочетание металлических и органических слоев может позволить совмещать эффек- 
тивную токовую накачку структуры и отвод тепла от активной области.

\section{Финансирование работы}

Работа поддержана Российским научным фондом (грант 21-12-00304).

\section{Конфликт интересов}

Авторы заявляют, что у них нет конфликта интересов.

\section{Список литературы}

[1] J.A. Lott, N.N. Ledentsov, V.M. Ustinov, N.A. Maleev, A.E. Zhukov, A.R. Kovsh, M.V. Maximov, B.V. Volovik, Zh.I. Alferov, D. Bimberg, Electron. Lett., 36, 1384 (2000). DOI: $10.1049 / \mathrm{el}: 20000988$

[2] F. Koyama, J. Lightwave Technol., 24, 4502 (2006). DOI: $10.1109 /$ JLT.2006.886064

[3] С.А. Блохин, М.А. Бобров, А.Г. Кузьменков, А.А. Блохин, А.П. Васильев, Ю.А. Гусева, М.М. Кулагина, Ю.М. Задиранов, Н.А. Малеев, И.И. Новиков, Л.Я. Карачинский, Н.Н. Леденцов, В.М. Устинов, Письма в ЖТФ, 44 (1), 67 (2018). DOI: 10.21883/PJTF.2018.01.45432.17029 [S.A. Blokhin, M.A. Bobrov, A.G. Kuz'menkov, A.A. Blokhin, A.P. Vasil'ev, Yu.A. Guseva, M.M. Kulagina, Yu.M. Zadiranov, N.A. Maleev, I.I. Novikov, L.Ya. Karachinsky, N.N. Ledentsov, V.M. Ustinov, Tech. Phys. Lett., 44 (1), 28 (2018). DOI: $10.1134 / \mathrm{S} 1063785018010042]$.

[4] M.A. Kaliteevski, A.A. Lazarenko, N.D. Il'inskaya, Yu.M. Zadiranov, M.E. Sasin, D. Zaitsev, V.A. Mazlin, P.N. Brunkov, S.I. Pavlov, A.Yu. Egorov, Plasmonics, 10, 281 (2014). DOI: 10.1007/s11468-014-9806-0

[5] G.M. Akselrod, E.R. Young, K.W. Stone, A. Palatnik, V. Bulović, Y.R. Tischler, Phys. Rev. B, 90, 035209 (2014). DOI: 10.1103/PhysRevB.90.035209

[6] R. Brückner, M. Sudzius, H. Fröb, V.G. Lyssenko, K. Leo, J. Appl. Phys., 109, 103116 (2011). DOI: 10.1063/1.3593188

[7] K.M. Morozov, P. Pander, L.G. Franca, A.V. Belonovski, E.I. Girshova, K.A. Ivanov, D.A. Livshits, N.V. Selenin, G. Pozina, A.P. Monkman, M.A. Kaliteevski, J. Phys. Chem. C, 125, 8376 (2021). DOI: 10.1021/acs.jpcc.1c02432

[8] K.M. Morozov, E.I. Girshova, A.R. Gubaidullin, K.A. Ivanov, G. Pozina, M.A. Kaliteevski, J. Phys.: Condens. Matter, 30, 435304 (2018). DOI: 10.1088/1361-648X/aae18c

[9] M. Koschorreck, R. Gehlhaar, V.G. Lyssenko, M. Swoboda, M. Hoffmann, K. Leo, Appl. Phys. Lett., 87, 181108 (2005). DOI: $10.1063 / 1.2125128$

[10] G. van Soest, A. Lagendijk, Phys. Rev. E, 65, 047601 (2002). DOI: 10.1103/PhysRevE.65.047601 